$$
\text { DRE/ER/45454 . TI }
$$

Annual Performance Report and Planned Research un

\title{
A STUDY OF TRANSIENT PARTICLE COARSENING
}

DOE/ER/45454--T1

DE93 005676

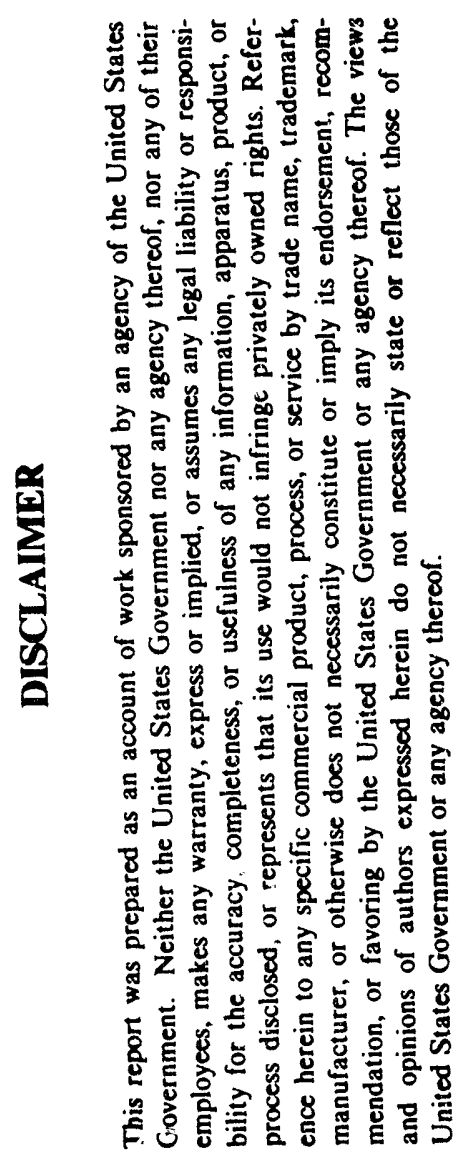

\author{
A program being conducted under \\ DOE Grant DE-FG06-91ER45454 \\ by \\ Jeffrey J. Hoyt \\ Principal Investigator
}

October 19,1992

Department of Mechanical and Materials Engineering

Washington State Lniversity

Pullman, Wa 9916t-2920 


\section{A Sudy of Transient Particie Coarsening}

The following sepresents a request for continuation of Department of Energ! contract no. DE-FG06-9/ER $45+5+$ for the perind $1 / 1 / 93-2 / 29 / 94$. Included in this dicument is a description of progress to date. a listing of personnel involied in the project, a list of other sources of external funding for the principal investigatur and a description of planned future work.

\section{Summary of Progress}

Over the past eighteen months we have concentrated our efforts on numerically modeling the time dependent particle coarsening process. The simulations have covered a time period from the point immediately after a quench into a two phase region until very late times where various asymptotic temporal laws are valid. Since the goal of our program is to compare experiments with various theories and decide which. if any; most accurately describe real systems. we have included four models in our numerical computations. The theories studied were: Lifshitz-Slyozor-Wagner (LSW) [1,2], Brailsford and Wynblatt (BIV) [3]. Marqusee and Ross (MR) [H] and Marder (MI) [5]. These particular descriptions of the Ostwald ripening process were chosen because they represent an increasing degree of mathematical sophistication: LSW is a mean field theory which is only valid at vanishing volu'me fraction of second phase particles, the BIV and WIR models include a correction for finite volume fraction effects and the Marder algorithm includes the effect of particle correlations. The simulations have monitored the particle size distribution as a function of time and, in order to be as realistic as possible, the initial PSD's have been derived from classical nucleation theory.

Figure 1 shows a the results of a typical simulation corresponding to a final volume fraction, $\phi_{f}$, of .01 . The quantities plotted are actually scaled variables; $\langle R\rangle$ the average radius is given by:

$$
\langle R\rangle=\frac{\langle r\rangle}{r_{0}^{*}}
$$

where $\langle r\rangle$ is the real average radius and $r_{0}^{*}$ is the initial critical radius (an input variable). Also, $t$ the scaled time is:

$$
t=\frac{D \phi_{f}}{r_{0}^{*}} t_{r}
$$

where $D$ is the diffusion coefficient and $\boldsymbol{t}_{\boldsymbol{r}}$ is the real time.

As seen in fig. 1 the dynamics of particle coarsening below a time of .1 is dominated by nucleation kinetics. At later times the four theories display a somewhat different kinetic behavior, but all eventually reproduce the well known $t^{\frac{1}{3}}$ power law. The mean field LSW theory predicts that the asymptotic regime is reached about an order of magnitude faster than the predictions of the Brailsford and Wynblatt or Marder models. Other than this observation, however, there is little to distinguish between the four curves of fig. 1 and we have tentatively concluded that the most reliable test of a given model is a study of the asymptotic behavior. This point will be further addressed below. 


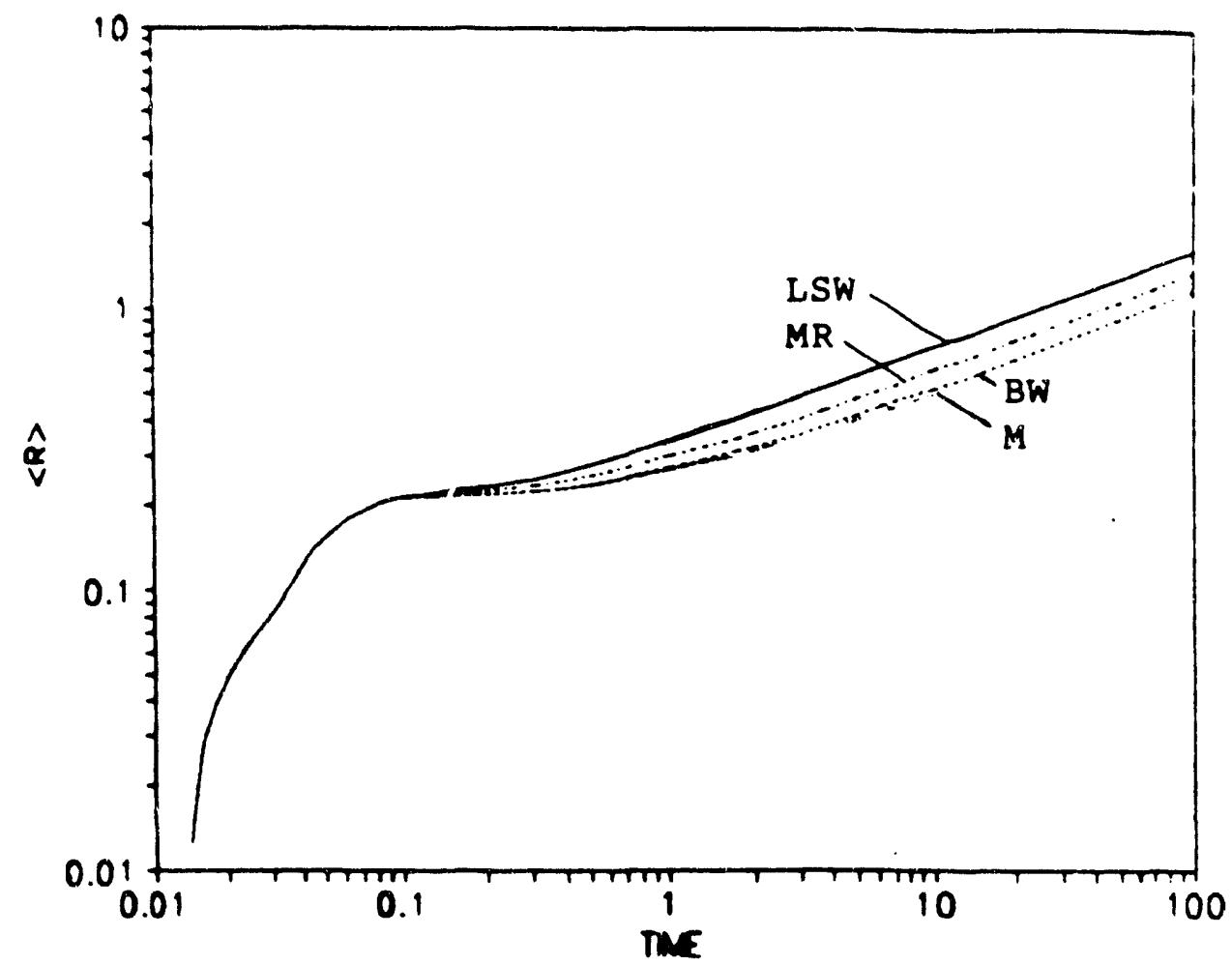

Figure 1. Scaled aresage radius vs. scaled time predicted by four coarsening theories. Volume fraction is .01 .

An alternative way of representing the ripening kinetics is a plot of the second moment of the normalized PSD vs. time (note the first moment is equal to unity). Figure 2 shows such a representation which corresponds to the same simulation shown in fig. 1 . The fact that all the curves in fig. 2 eventually reach a constant value indicates that each model predicts a scaled PSD which acts as a unique attractor state for the nonlinear problem.

In comparing figs. 1 and 2 one can conclude that, for any of the four models investigated, the $t^{\frac{1}{3}}$ power law behavior sets in much earlier than the attainment of a time independent scaled PSD. For example, in the LSW result, the curve in fig. 1 becomes linear at a time of about 1.0 whereas a significant leveling off of the data in fig. 2 occurs at a time of approximately 50.0. The above observation is extremely important when interpreting experimental data; that is, one can not use a measure of the average particle radius is. time as a guide when defining an asymptotic PSD.

The numerical simulations described briefly above represent the topic of a presentation to be delivered at a symposium entitled "Modeling of Coarsening and Grain Growth", TMS Fall Meeting, Chicago (Nov. 1992). The results will also be prepared for publication in the near future.

The transient coarsening behavior, although very important to the overall understanding of the phenomenon, can not yield information as to which model of Ostwald ripening is most accurate. Indeed the key difference in the four plots of fig. 1 occurs in the asymptotic regime. Thus, a major nagging question in coarsening theory remains unsolved; namely, why does the theoretically predicted and the experimentally determined time independent scaled PSD's consistently disagree? Experimentally measured size distributions always appear broader than those calculated by any modern coarsening theory. 


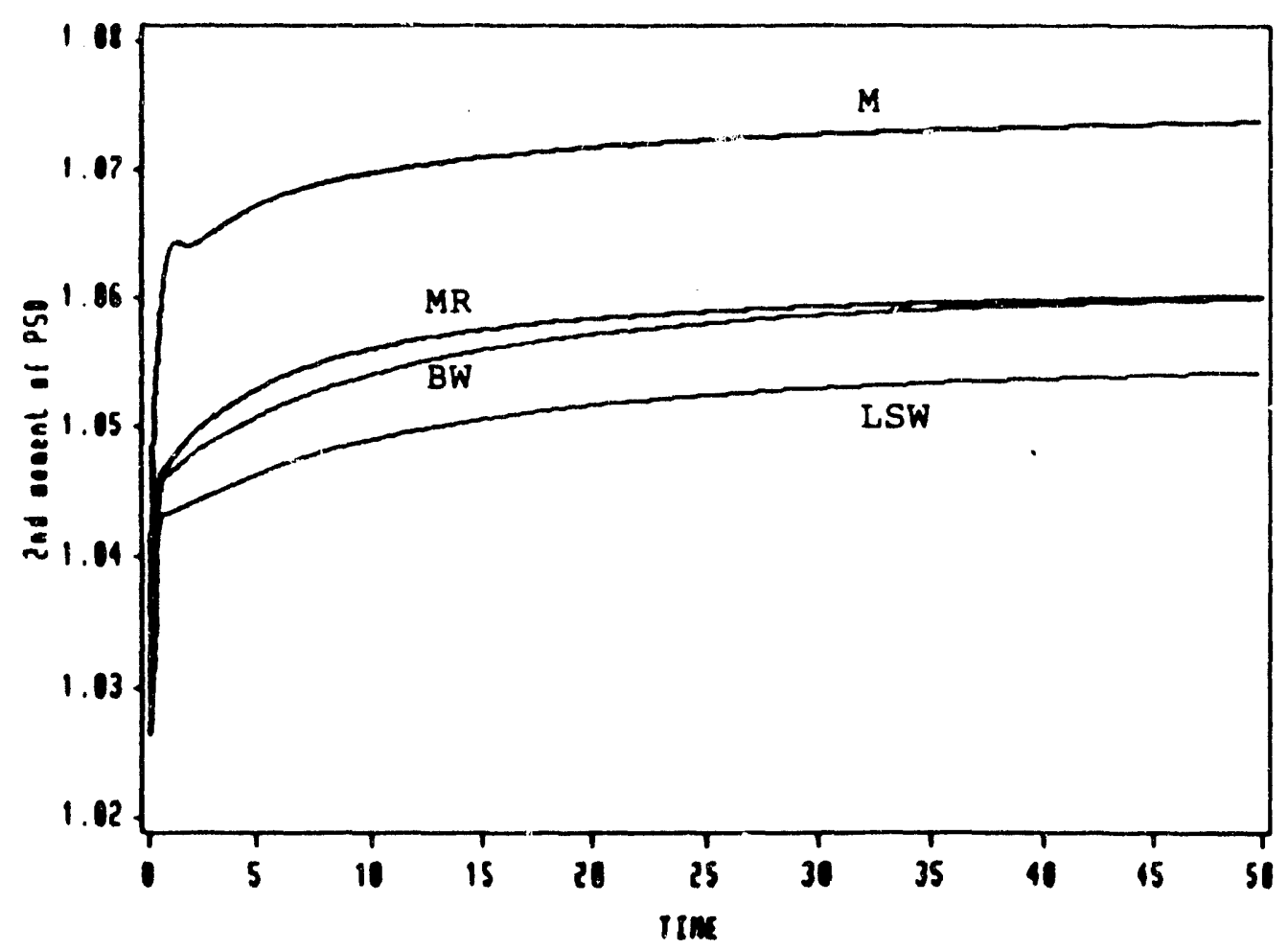

Figure 2. The second moment of the scaled particle size distribution vs. scaled time, $\phi_{f}=.01$

We have recently formulated a geometric correction factor which has been neglected in most previous experimental studies and which must be applied to experimental data in order to claim a valid comparison to theory. This factor will be briefly discussed below.

The only reliable method of obtaining PSD's is via the use of transmission electron microscopy (TE.M). The shaded area of fig. 3 represents the cross section of a TEM thin foil, ie. the electron beam in this schematic drawing travels from left to right, and the circles in the diagram represent spherical particles. All paticles whose centers lie in the shaded region, for example those labelled A, B and C, will be projected at their true size in the subsequent TEM photomicrograph. However, the important point to note is that those precipitates whose centers were located in bulk material which had been removed during specimen preparation ( $D, E$, and $F$ ) will be recorded at less than actual size.

Consider, then, the consequences of the geometric factor depicted in fig. 3. Clearly measurements using TEM will yield particle sizes which are less than or equal to the actual sizes. As a result the measured particle size distribution will be skewed toward smaller values of $r$ and the average, $\langle r\rangle$, will be less than the true average. Since a comparison of theory and experiment requires the PSD to be expressed on a normalized scale, ie. $r /\langle r\rangle$, neglect of the aforementioned geometric factor implies ar measured PSD which is always broader than the true distribution.

It is a strightforward mathematical exercise to correct the measured PSD for the geometric effect shown in fig. 3 and preliminary simulations of corrected vs. uncorrected PSD's have indicated that the effect is by no means trivial. (It should be noted that the error in the measured average radius will have little affect on $\log -\log$ plots of $\langle r\rangle$ vs. aging time.) We feel that the correction may be an important factor in establishing a 


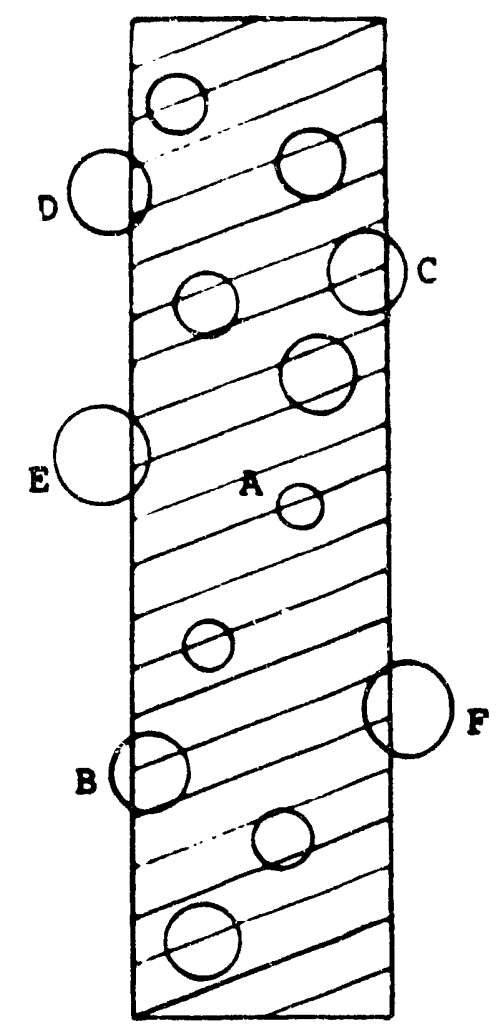

Figure 3. Schematic drawing of a cross section of a TE.I thin foil showing the positions of various spherical precipitates.

closure between experiment and the theory of particle coarsening and as such we plan to publish a short letter on this subject.

\section{Personnel}

The particle coarsening study is currently supporting one graduate student, Mr. D. Che, who is basing his $\mathrm{PhD}$ dissertation on the theoretical and experimental results. He expects to complete the degree in approximately $2 \frac{1}{2}$ years. The experimental portion of the research involving small angle $x$-ray scattering will be conducted in close collaboration with Dr. S. Spooner of the Sulid State Division at the Oak Ridge National Laboratory. The principal investigator on this work is J. J. Hoyt.

\section{Other Funding Sources}

DOE - "A Study of Transient Particle Coarsening," (DE-FG06-91ER45454) This request.

NSF - "Nucleation and Growth in Al-Zn and Order-Disorder in $\mathrm{AgAuCd}_{2}$, " (DMR 8919193), $\$ 247,700,5 / 1 / 90$ - 10/31/93. Renewal submitted 10/19/92. 
Planned Research During Second Budget Period

In the upcoming year we plan to focus our efforts on collecting kinetic data of particle coarsening in the Al-Li system. A crucible furnace, salt pot. TE.M jet polisher and Al-Li alloys of three different average concentrations (7,9 and 11 at $\%$ ) have been obtained. We are currently investigating various heat treatment schedules and will have initial TE.I results during the upcoming spring semester. A key ingredient in the experimental work is the use of both TE.I and small angle x-ray scattering (S.AXS) on the same samples. The use of both techniques simultaneously will provide accurate measures of various features of the PSD. The SAXS studies will be performed at the $10 \mathrm{~m}$ facility located at the Oak Ridge National Laboratory and our first trip is tentatively scheduled for the end of spring: semester.

Two new developments in particle coarsening theory will influence the interpretation of our Al-Li data. The first is the geometric correction alluded to in the first section of this report. We hope to show that properly corrected PSD's in fact show good agreement with theory. The second development is the formulation of improved coarsening theories by Marsh [6] and Akaiwa and Voorhees [7]. These two theories are, in a sense, exact in that an ensemble of particles is established and the growth of each particle is determined by the flux of solute to the buundary. Any approximations due to the statistical averaging of particles, as is found in previous theories, are avoided entirely. The two new contributions are able to monitor particle correlations as a function of time and, as a result, are able to predict SAXS spectra directly. Thus, our scattering results will form the basis for an interesting and informative comparison with the most sophisticated models of coarsening phenomena. 


\section{References}

1. I. M. Lifshitz and I. I. Slỵozor, J. Chem. Phys. Solıds 19. 35 (1961).

2. C. Ilagner, Z. Electrochem., 65. 591 (1961).

3. A. D. Brailsford and P. Wynblatt. Acta Metall., 27. 489 (1979).

4. J. . Marqusee and Russ: J. Chem. Phys., 80. 536 (1980).

5. M. Marder, Phys. Rev., A36. 858 (1987).

6. S. P. Marsh, PhD dissertation. Rensselear Polytechnic Institute. Troy. NY' (1989).

7. N. Akaiwa and P. II. Voorhees. Proceedings of T.MS symposium ". Hodeling of Coarsening and Grain Growth," T.MS Annual Meeting. Chicagu, IL (1992).

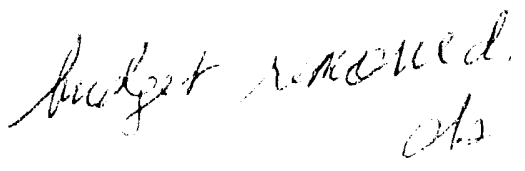



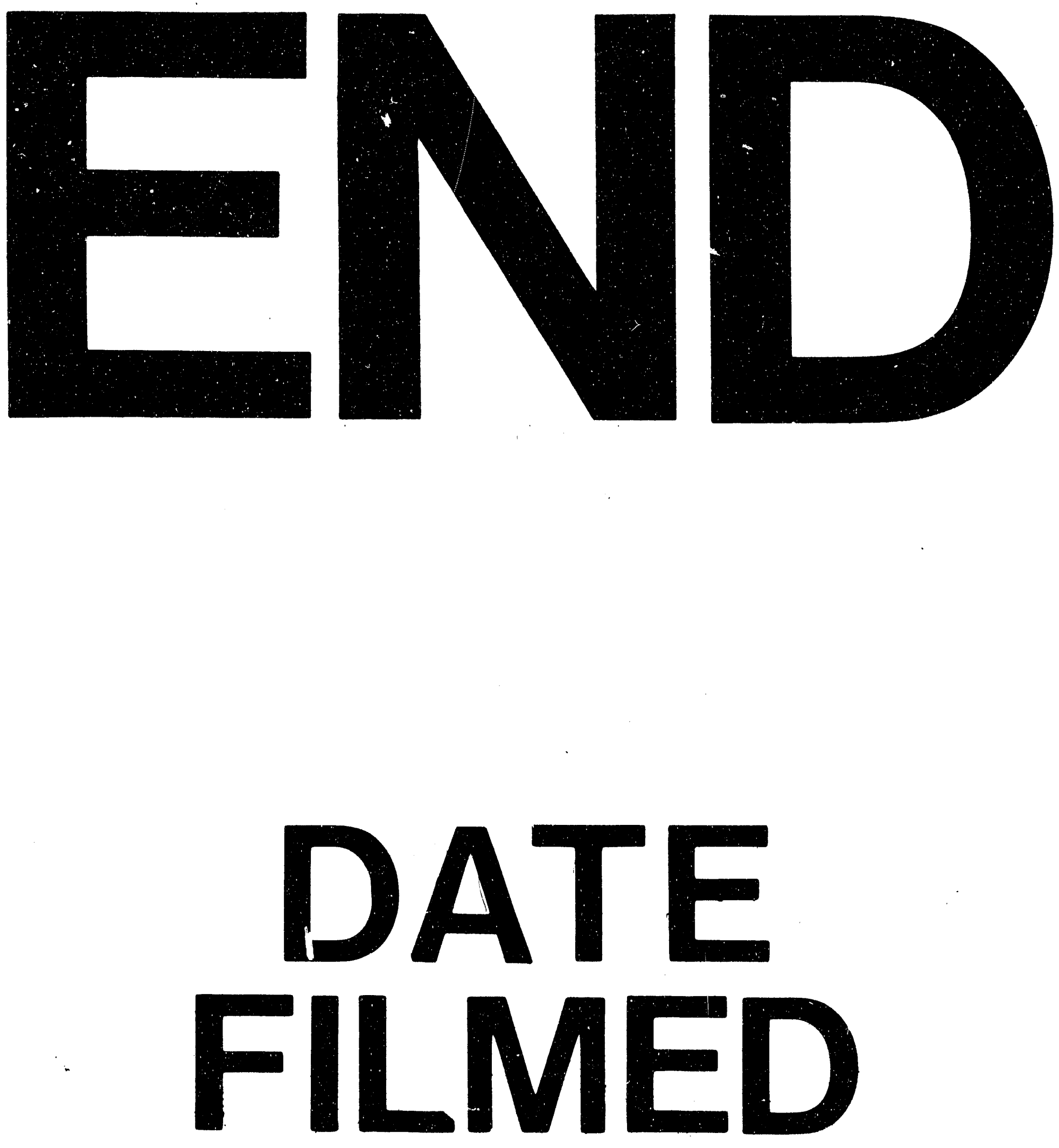

I

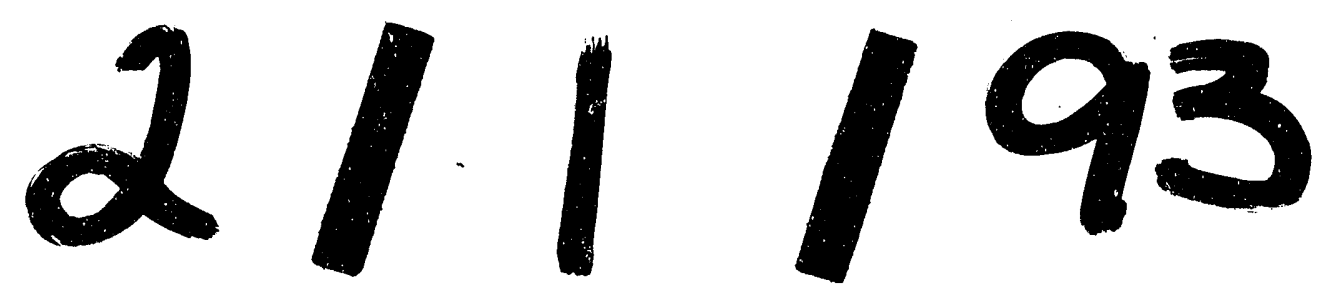


